\title{
Ultrafast, Optical Parametric Chirped-pulse Amplification System using a Block of Transparent Material for Pulse Stretching and Compression
}

\author{
Koichi Yamakawa $^{* 12}$, Yutaka Akahane ${ }^{* 12}$ and Kanade Ogawa ${ }^{* 12}$ \\ ${ }^{* 1}$ Japan Atomic Energy Agency, 8-1-7 Umemidai, Kizugawa, Kyoto 619-0215, Japan \\ E-mail: yamakawa.koichi@jaea.go.jp \\ *2 CREST, Japan Science and Technology Agency, Sanbancho 5, Chiyoda-ku,, Tokyo 102-0075, Ja- \\ pan
}

\begin{abstract}
We have proposed and demonstrated a very simple and robust femtosecond optical-parametric chirped-pulse amplification scheme in which an even order dispersion of an idler pulse is compensated by passing through an identical positive dispersive material used for temporal stretching a signal pulse. By compressing the idler pulses having a negative chirp in this manner, high power sub100 fs pulses were successfully obtained with only a transparent glass block used for the stretcher and compressor.
\end{abstract}

DOI:10.2961/jlmn.2012.03.0015

Keywords: optical parametric chirped-pulse amplification, idler pulse, micro-machining, Yb:YLF, glass block

\section{Introduction}

During the last two decades, there has been a great progress in high peak-power short-pulse laser development. Since the invention of chirped-pulse amplification (CPA), duration of the intense laser pulses has been reduced from picosecond to femtosecond regime, which makes it possible to construct table-top high-power ultrashort pulse lasers in user laboratories [1]. Intense ultrashort pulses are now widely used in various applications ranging from ultrafast spectroscopy, imaging and material processing to highfield sciences such as attosecond high-order harmonics generation and high energy particle beam generation and inertial confinement fusion [2]. In the point of view of real world industrial applications such as precise micromachining and fabrication, however, the ultrafast CPA laser have not been established as conventional high-power $\mathrm{Nd}: \mathrm{YAG}$ or $\mathrm{CO}_{2}$ lasers. There is an interesting feature in ultrafast laser ablation that laser energy is deposited with shorter time than thermal relaxation of materials. The shorter-time energy deposition prevents molten material on metals as well as heat-affected zones in transparent materials, which will boost ultrafast lasers to essential industrial tools for fabrication of precise microstructures on material $[3,4]$.

The main reason of CPA lasers holding a minority position in industries lies in the weakness of the laser system. CPA lasers always have pulse stretching and compression processes for safe amplification. Seed pulses from an ultrashort pulse oscillator are temporally stretched by a gratingbased stretcher or single-mode optical fiber before amplification to avoid damage of gain media and optical components during amplification. Amplified pulses are then sent to the negative-dispersive line such as grating or prism pairs and substantially recompressed. Optics used for the pulse stretcher and/or compressor, however, are so delicate that it should be handled with great care. Dispersive com- ponents consisted of these optics are very sensitive to temperature variation and misalignment, which can introduce pulse broadening after compression. Furthermore input-tooutput optical efficiencies of these dispersive components are usually low, $\sim 50 \%$ for the grating pair, for example, which losses about a half of amplified outputs. To overcome these drawback, simple, robust and efficient CPA laser are needed.

Several approaches have been made for constructing practical ultrafast CPA lasers. Ytterbium (Yb)-doped fiber CPA lasers are particularly interesting due to good thermooptical properties and excellent output beam quality with picosecond or sub-picosecond durations and high average powers of more than $100 \mathrm{~W}$ at high repetition rates $[5,6]$. The gain bandwidth of the amplification medium is, however, not enough to generate ultrashort pulses as we can be obtained with Ti:sapphire. Alternatively Gaudiosi and coworkers have constructed $11 \mathrm{~W}, 100 \mathrm{kHz}$ Ti:sapphire CPA laser system with down chirped pulse amplification (DPA) scheme [7] The seed pulse has been temporally expanded by negative chirp, and the amplified pulse has been recompressed to 28 fs with high efficiency by passing through the highly-dispersive glass blocks. But both grating and prism pairs are necessary as a negative stretcher for precise dispersion management including large amount of materials in the Ti:sapphire amplifier stage.

In this research, we have introduced optical-parametric chirped-pulse amplification (OPCPA) technique $[8,9]$ for constructing practical femtosecond CPA laser, which employs optical-parametric amplification (OPA) as high-gain amplification stages in CPA. The OPA is a direct energytransfer from pump to signal pulses accompanied by the generation of the idler pulses in the parametric process. Due to the phase-conjugation process, the sign of the even orders of spectral dispersion of the idler pulse is inverted from the signal [10-12], which the even orders of spectral 
dispersion can be compensated by passing through the same positive-dispersive material as the pulse stretcher. Indeed, with compression of the idler pulse, high-power ultrashort laser pulse with 74 fs duration in full width at half maximum (FWHM) was successfully obtained using an identical glass block as pulse stretcher and compressor in our experiment. This scheme is very attractive for the construction of the simple, robust, and efficient ultrafast OPCPA lasers, since the dispersive medium is compact, solid and lossless.

\section{Theory}

The theoretical descriptions of temporal phase in OPCPA have been made by Ross and co-workers [13]. The spectral phase of the idler pulse $\phi_{i}\left(\omega_{i}\right)$ is determined by initial spectral phase of pump and signal pulses, as described below,

$$
\phi_{i}\left(\omega_{i}\right)=\phi_{p}\left(\omega_{p}\right)-\phi_{s}\left(\omega_{s}\right)-\frac{\pi}{2}-\frac{\Delta k L}{2}
$$

whrere $p, s$, and $i$ suffixes mean pump, signal and idler pulses, respectively, $\Delta k$ is the phase mismatch and $\mathrm{L}$ is the interaction length in OPA proess. For the energy conservation law, there is a general relationship among frequencies of pump, signal and idler pulses at each moment,

$$
m^{b}=m^{2}+m^{!}
$$

On assumption of constant pump phase and negligible phase mismatch term, the group-delay dispersion (GDD), which is second-order differential of eq. (1), is described as follows with substitution of eq. (2).

$$
\psi_{i}\left(\omega_{i}\right)=-\not\left(\omega_{s}\right)
$$

As seen in eq. (3), the GDD of generated idler pulse in OPA process is opposite sign of the signal pulse, which means the temporal chirp of the idler pulse is inverted from the signal. Piskarskas and co-workers firstly reported this phenomena as "chirp reversal" [10]. As the same as GDD, signs of the even orders of spectral dispersion of the idler pulse are found to be inverted from the signal by considering higher-order differentials of eq. (1). Inverted even orders of spectral dispersions can be compensated by passing through the same dispersive component as the pulse stretcher. When the transparent material such as a highlydispersive glass block is used for producing positively chirp of the signal pulse before amplification, the idler pulse having a negative chirp can be simply compressed by passing through the same material.

While even order dispersions are compensated, odd order dispersions are conserved and accumulated on pulse expansion and compression in the scheme. To evaluate an effect of residual higher odd order dispersions, we have made simple calculation of the temporal shape of compressed idler pulse. Figure 1 shows a calculation result of the idler pulse compression with this scheme using an identical glass block. The center wavelength and duration of seed signal pulse are $1020 \mathrm{~nm}$ and $59 \mathrm{fs}$ (FWHM), respectively. The glass block is made of SF11 highly-dispersive glass block with $75 \mathrm{~cm}$ long. On assumption that even orders of spectral phase of idler pulse are successfully compensated, duration of the compressed idler pulse is $80.5 \mathrm{fs}$ (FWHM). Although there are several post pulses caused by residual odd order dispersions, such as $1.8 \times 10^{5} \mathrm{fs}^{3}$ of third order, the energy contents in he main pulse is $89 \%$. From these calculation results, our scheme is effective enough to generate sub-100fs intense laser pulse when we compress 5 ps idler pulses.

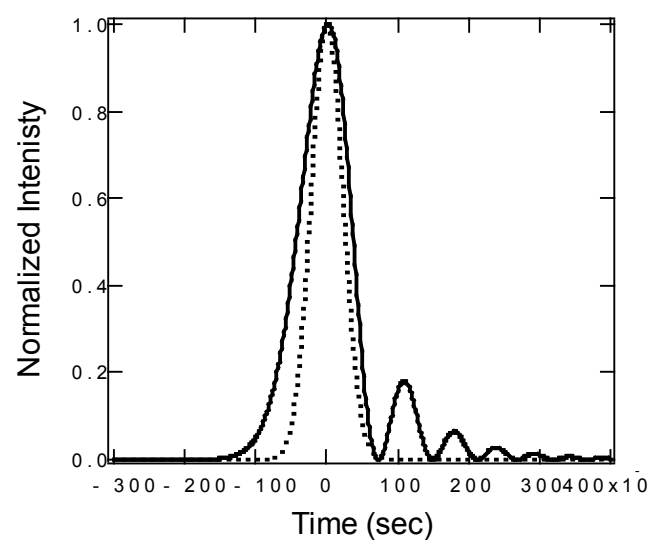

Fig. 1 Tempporal profile of compressed idler pulse with $75 \mathrm{~cm}$ SF 11 glass block for pulse stretcher and compressor (solid-line, calculation). The duration of the compressed pulse is $80.5 \mathrm{fs}$. Dotted line is the Fourier transform-limited pulse (59 fs).

\section{Experimental setup}

The experimental setup is shown in Fig.2. An output pulse with $1020 \mathrm{~nm}$ center wavelength from a mode-locked Ti:sapphire oscillator (Spectra Physics:TSUNAMI) was split into two beams. Each beam was used as the seed pulse for the Yb:YLF CPA pump laser [14, 15] and OPCPA, respectively. Seed pulse of Yb:YLF CPA pump laser was temporally stretched to $1.2 \mathrm{~ns}$ by $1.2 \mathrm{~km}$ polarizationmaintained single-mode fiber (Fujkura: PANDA fiber SM98-PS-U25A) before amplification. Amplified output pulse from the $\mathrm{Yb}$ :YLF regenerative amplifier was compressed by a grating-pair based pulse compressor to $\sim 2 \mathrm{ps}$. The compressed output was frequency-doubled and downcollimated to $1 \mathrm{~mm}$ in diameter and injected to the OPA crystal (Type-I BBO, 7mmt). The energy of the OPA pump pulse before OPA crystal was $0.9 \mathrm{~mJ}$. The seed signal of OPCPA was temporally stretched by the highly-dispersive glass block (SCHOTT SF11, 50mmt) and also collimated to $1.1 \mathrm{~mm}$ in diameter. An internal crossing angle was set to be 1.2 deg between pump and seed signal pulses. The repetition rate of the OPA stage was $10 \mathrm{~Hz}$, which was limited by the pump laser. In OPA stage, typical gain of $3 \times 10^{3}$ which corresponding to $\sim 6 \mu \mathrm{J}$ of signal pulse energy, was obtained with a pump intensity of $81 \mathrm{GW} / \mathrm{cm}^{2}$. The idler pulse generated in the OPA crystal was collimated by spherical mirrors and sent back to the identical SF11 glass block for pulse compression. The transmittance of the glass block was limited to $86 \%$ due to Fresnel reflection losses on input and output optical surfaces in this experiment. With anti-reflection coating on these surfaces the glass block has no attenuation during compression. The energy 
of the idler pulse was measured to be $\sim 6.4 \mu \mathrm{J}$ by pyroelectric detector (OPHIR PE-10). After passing through the glass block, the duration of the idler pulse was measured by a single-shot second harmonic generation ( $\mathrm{SHG}$ ) autocorrelator.

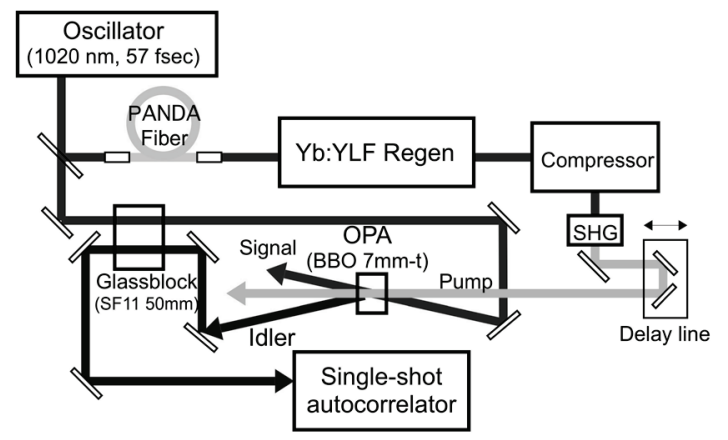

Fig. 2 Experimental setup.

\section{Results and discussion}

Figure 3 shows autocorrelation traces of the seed signal pulse from the mode-locked oscillator and after the glassblock stretcher, respectively. The pulse duration of the original seed pulse was 57 fs (FWHM) assuming a $\operatorname{sech}^{2}$ temporal profile, which is close to the Fourier transformlimited ones $(51.7 \mathrm{fs})$. The duration of the seed pulse was stretched to $390 \mathrm{fs}$ (FWHM) after passing through the glass block. A pulse duration mismatch between seed (390 fs) and pump ( 2 ps) is considered to be caused by lower OPA gain and efficiency in this experiment.

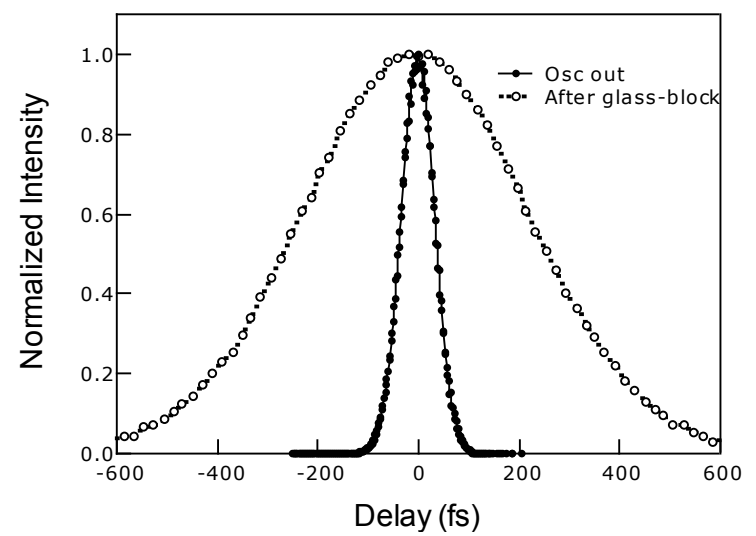

Fig. 3 Autocorrelation traces of seed signal pulses. Autocorrelation widths of signal pulse without (solid line and closed circles) and with (dashed line and open circles) glass block were $80 \mathrm{fs}$ and $550 \mathrm{fs}$, respectively, which corresponds to $57 \mathrm{fs}$ and $390 \mathrm{fs}$ pulse duration assuming a $\operatorname{sech}^{2}$ temporal profile respectively.

Typical OPA spectra of the signal and idler pulses are shown in Fig. 4. The center wavelength of the idler pulse was shifted to $\sim 40 \mathrm{~nm}$ with respect to the signal one. This is due to the non-collinear geometry of OPA in the experimental setup. Measured autocorrelation traces of the un- compressed and compressed idler pulses are also shown in Fig. 5. Whereas the duration of the uncompressed pulse was 295 fs (FWHM), the compressed idler pulse was 73.9 fs (FWHM). The pulse duration of the compressed pulse is slightly longer than the Fourier-transform limited one (59.3 fs), which may be due to the spectral mismatch between the signal and idler pulses as seen in Fig.4. The group-delay dispersion (GDD) of the glass block at $980 \mathrm{~nm}$ is $6812 \mathrm{fs}^{2}$, which is not significantly different from the GGD at 1020 $\mathrm{nm}\left(6376 \mathrm{fs}^{2}\right)$. Therefore it does not cause notable pulse broadening of sub-100 fs pulses in this experiment. In a nearly degenerate OPA geometry, the spectral mismatch between signal and idler pulses becomes negligible.

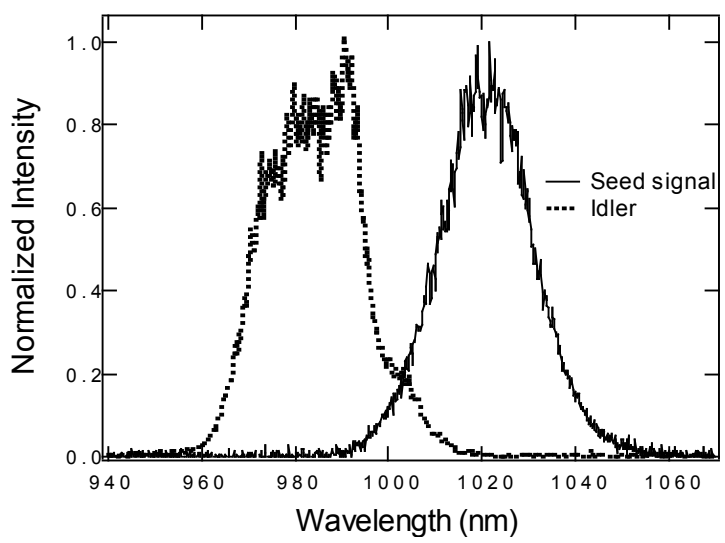

Fig. 4 OPA spectra of signal (solid line) and idler (dashed line) pulses. The center wavelength of the idler pulse was shifted to $\sim 40 \mathrm{~nm}$ with respect to the signal pulse. FWHM bandwidth of the signal and idler pulses are $22 \mathrm{~nm}$ and $26 \mathrm{~nm}$, respectively.

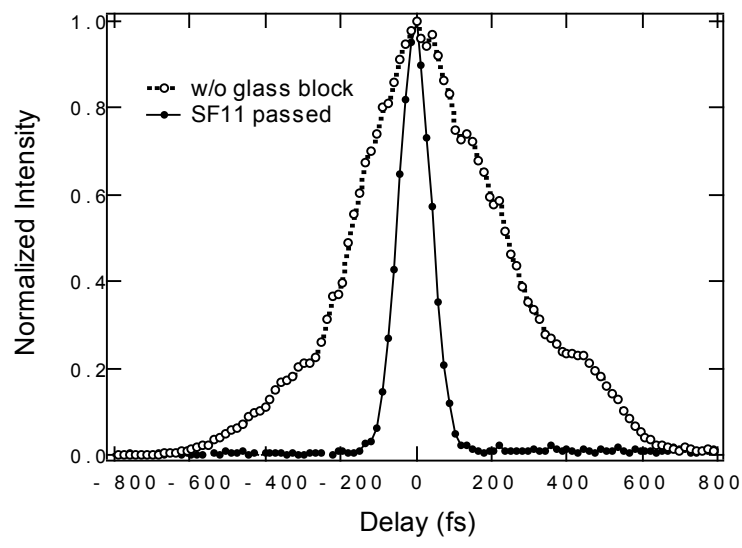

Fig. 5 Autocorrelation traces of idler pulses. Autocorrelation widths with (solid line and closed circles) and without (dased line and open circles) SF11 glass block were measured to be $104 \mathrm{fs}$ and $416 \mathrm{fs}$, respectively. Pulse durations of the idler pulses were 73.9 and $295 \mathrm{fs}$, respectively.

It should be mentioned that an idler pulse is known to have angular spatial dispersion on non-colliner OPA. There are some serious effects from the angular dispersion on propagating and focusing laser pulses in OPCPA with Ti:Sapphire laser, which prevents idler pulses from applications. On the other hand, our OPA is near collinear geometry and the angular divergence of the idler pulse in our case is calculated to be $760 \mu \mathrm{rad}$., which is so small that the 
idler pulse can be handled by normal spherical or cylindrical mirrors. Indeed, no effect of the angular dispersion was observed in our experiments.

\section{Prospects}

Since successful idler pulse compression has been demonstrated using the identical positive stretcher/compressor combination in OPCPA, the ideal ultrafast OPCPA systems employing this scheme can be designed for the real world applications in future. Instead of the Ti;sapphire mode-locked oscillator and Yb:YLF CPA laser, more reliable Yttrium-doped sub-100 fs fiber oscillator [16] and fiber amplifier operating at $1030 \mathrm{~nm}$ center wavelength can be used as seed and pump sources, respectively. Amplified output pulses from the fiber amplifier are compressed to $\sim 10$ ps without CPA due to gain narrowing and then frequency-doubling before OPA stage. Seed pulses for OPCPA are temporally stretched to $\sim 5$ ps by passing through the SF11 glass block (total pass length: $75 \mathrm{~cm}$ ) for efficient OPA and injected to the OPA crystal in coincidence with pump pulses. The OPA stage can be double-pass configuration with almost collinear geometry (crossing angle $\sim 0.3$ deg) where signal and pump pulses are spatio-temporally overlapped twice in the OPA crystal. On assumption of $\sim 1 \mathrm{~mJ}$ pump energy, energetic idler pulses of $\sim 300 \mu \mathrm{J}$ energy will be generated considering the depression of pump intensity during OPA. The idler pulse is then compressed without losses using the same glass block as the pulse stretcher, in which sub-100 fs, sub-mJ class laser pulse should be obtained. The repetition rate of the laser system mainly depends on the pump laser, as high as few $\mathrm{MHz}$, which corresponds to an average power of more than 300 $\mathrm{W}$, is available with current fiber-laser technologies [17]. There is another merit with this scheme. Signal pulses are also amplified up to the same energy as idler. The duration of the amplified signal pulse is $\sim 5$ ps and this pulse is available for rough-premachining, which will help to reduce the processing time in the laser-machining. This kind of simple, robust and versatile laser tools with high peak and high average powers will play an important role in

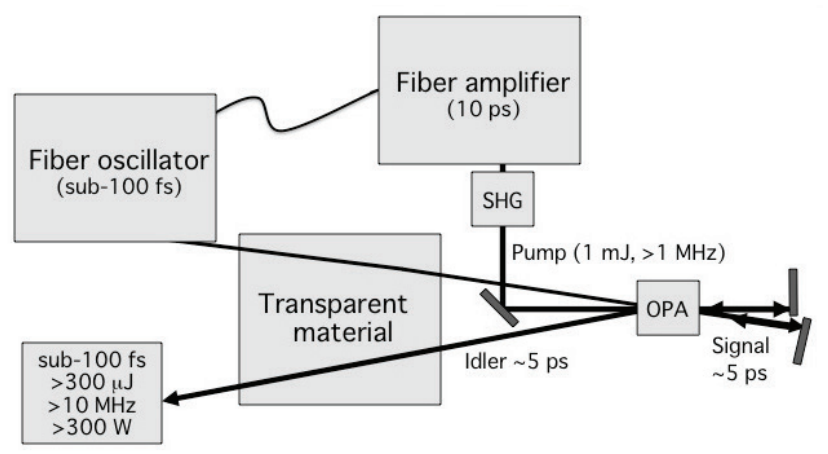

Fig. 6 Real world high-power femtosecond laser system. factories in the near future.

\section{Conclusion}

Compression of the idler pulse in femtosecond OPCPA with an identical glass block as both pulse stretcher and compressor has been proposed and demonstrated. Successful compressed pulse with sub-100fs duration was obtained in the experiment. With this scheme a simple, robust and efficient femtosecond OPCPA laser can be constructed, which is useful for industrials such as material processing as well as other widespread applications.

\section{References}

[1] K. Yamakawa and C. P. J. Barty: IEEE J. Sel. Top. Quantum Electron. 6 (2000) 658. (Journals)

[2] M. D. Perry and G. Mourou: Science 264 (1994) 917. (Journals)

[3] X. Liu, D. Du and G. Mourou: IEEE J. Quantum Electron. 33 (1997) 1706. (Journals)

[4] D. C. Deshpande, A. P. Malshe, E. A. Stach, V. Radmilovic, D. Alexander, D. Doerr, and D. Hirt, D: J. Appl. Phys. 97 (2005) 074316. (Journals)

[5] L. Shah, Z. Liu, I. Hartl, G. Imeshev, G. C. Cho and M. E. Fermann: Opt. Expres. 13 (2005) 4717. (Journals)

[6] F. Röser, T. Eidam, J. Rothhaardt, O. Schmidt, D. N. Schimpf, J. Limpert and A. Tunnermann: Opt. Lett. 32 (2007) 3495. (Journals)

[7] D. M. Gaudiosi, A. L. Lytle, P. Kohl, M. M. Murnane, H. C. Kapteyn and S. Backus: Opt. Lett. 29 (2004) 2665. (Journals)

[8] I. N. Ross, P. Matousek, M. Towrie, A. J. Langly and J. L. Collier: Opt. Commun. 144 (1997) 125. (Journals)

[9] A. Dubietis, R. Butkus and A. P. Piskarskas: IEEE J. Sel. Top. Quantum Electron. 12 (2006) 163. (Journals)

[10]A. Piskarskas, A. Stabinis and A. Yankauskas: Sov. Phys. Usp. 29 (1986) 869. (Journals)

[11]H-S. Tan, E. Schreiber and W. S. Warren: Opt. Lett. 27 (2002) 439. (Journals)

[12]T. Witte K. L. Kompa and M. Motzkus: Appl. Phys. B76 (2003) 467. (Journals)

[13] I. N. Ross, P. Matousek, G. H. C. New and K. Osvay: J. Opt. Soc. Am. B19 (2002) 2945. (Journals)

[14]J. Kawanaka, K. Yamakawa, H. Nishioka and K. Ueda: Opt. Lett. 28 (2003) 2121. (Journals)

[15]K. Yamakawa, M. Aoyama, Y. Akahane, K. Ogawa, K. Tsuji, A. Sugiyama, T. Harimoto, J. Kawanaka, H. Nishioka, and M. Fujita: Opt. Expres. 15 (2007) 5018. (Journals)

[16] F. O. Ilday, J. Buckley, R. H. Lim, F. W. Wise and W. G. Clark: Opt. Lett. 28 (2003) 1365. (Journals)

[17] T. Eidam, S. Hadrich, F. Röser, E. Seise, T. Gottschall, J. Rothhardt, T. Schreiber, J. Limpert and A. Tunnermann: IEEE J. Set. Top. Quamtum Electron. 15 (2009) 187. (Journals) 